\title{
Discerning between Ambiguity and Ambivalence Using Range Responses
}

\author{
Daniel J. Rozell \\ Department of Technology and Society, Stony Brook University, USA \\ daniel.rozell@stonybrook.edu
}

8 January 2022

\begin{abstract}
:
This brief note introduces the use of a range of responses rather than a single response for an opinion survey question. The methodological advantage of collecting this additional information is that it can help discern between ambiguity and ambivalence in situations where it is valuable to further investigate the nature of a respondent's indecision.
\end{abstract}

Keywords: social statistics, surveys, public opinion, risk perception

In surveys, selecting the neutral or "neither agree nor disagree" Likert scale option can be alternately interpreted as either ambiguity ("I don't know”) or ambivalence ("I don't care”) (e.g., Baka, Figgou, and Triga 2012). Even in continuous scale data, such as subjective probability estimates of future events, there is a bias towards the middle value (Hurd 2009) which is likewise difficult to interpret (Bruine de Bruin and Carman 2012; 2018). Furthermore, adding a "Don't Know" option to resolve this confusion may lower data quality by encouraging respondents to avoid the effort of rendering an opinion (Krosnick et al. 2002).

One potential method for resolving this lack of clarity in middle-value responses is to use a range of values to represent a single response. The term "interval" to represent range-response survey data is avoided as the term is already used to define data with equidistant scale points as distinguished from nominal, ordinal, or ratio scale data (e.g., Allen and Seaman 2007). Rangeresponse data were perhaps avoided in the past due to computational burden, but the statistical analysis of such data is now possible due to developments in the field of interval analysis (e.g., Ferson et al. 2007). Likewise, collecting range responses requires additional work in that both a lower and upper bound response is required, but the advantage is the ability capture the uncertainty within responses and not just the variation among responses. 
In practice, a range response includes two points that encompass the respondent's range of beliefs. The size of the range corresponds to the respondent's confidence in the belief. That is, if a respondent gives a single response to a standard survey question, we generally assume that the value represents the respondent's opinion at that moment (unless there are reasons to believe they are not being honest). Likewise, if a respondent can give a range of values to represent their opinion, it should hold that the range also represents their opinion at that moment. If the respondent has a clear opinion, it would be expressed as a single choice or a narrow range. Conversely, if the respondent is uncertain about their opinion, it would be expressed as a wide range - the wider the range, the less confidence the respondent has in their opinion. A small range only means ambivalence if it is centered around the neutral response. A narrow range at either end of the scale stills signals agreement or disagreement, but we are using the idea of range responses here to explore the multiple interpretations of the neutral opinion response.

For example, if a question asked for the likelihood of an event on a continuous scale, a selected range of $45 \%$ to $55 \%$ suggests that the respondent actually believes the likelihood is about $50 \%$, whereas a range of $1 \%$ to $99 \%$ indicates true incertitude that might otherwise show up as a 50\% response if only one number could be selected. Similarly, selecting a range of 4 to 6 on a 9-point Likert-type scale suggests ambivalence whereas selecting the range 1 to 9 indicates ambiguity.

One could argue that the ambiguity/ambivalence distinction is not always important, but some major events, such as elections, are frequently decided by weakly committed survey respondents. Determining the nature of these middle opinions can be valuable information. For example, different approaches may be required to influence voters who have ambiguity based on a lack of knowledge compared to voters who have ambivalence based on perceived conflicting personal values (e.g., freedom vs. security). Likewise, the additional information in range responses can help signal to survey authors when a question is too vague if most respondents show broad ambiguity. 
As a final word of caution, it is well known that people frequently suffer from overconfidence or certainty bias regarding objective information and are prone to give a narrower range to express their uncertainty than is warranted (Fischhoff, Slovic, and Lichtenstein 1977). Thus, range responses are better used for opinion polling than estimating objective information or expert elicitation of probabilities for future events.

\section{References}

Allen, I. Elaine, and Christopher A. Seaman. 2007. "Likert Scales and Data Analyses." Quality Progress 40 (7): 64-65.

Baka, Aphrodite, Lia Figgou, and Vasiliki Triga. 2012. "“Neither Agree, nor Disagree': A Critical Analysis of the Middle Answer Category in Voting Advice Applications." International Journal of Electronic Governance 5 (3-4): 244-63. https://doi.org/10.1504/IJEG.2012.051306.

Bruine de Bruin, Wändi, and Katherine G. Carman. 2012. "Measuring Risk Perceptions: What Does the Excessive Use of 50\% Mean?” Medical Decision Making 32 (2): 232-36. https://doi.org/10.1177/0272989X11404077.

—. 2018. "Measuring Subjective Probabilities: The Effect of Response Mode on the Use of Focal Responses, Validity, and Respondents' Evaluations.” Risk Analysis 38 (10): 212843. https://doi.org/10.1111/risa.13138.

Ferson, Scott, Vladik Kreinovich, Janos Hajagos, William Oberkampf, and Lev Ginzburg. 2007. "Experimental Uncertainty Estimation and Statistics for Data Having Interval Uncertainty. SAND2007-0939. Sandia National Laboratories.” Albuquerque, New Mexico.

Fischhoff, Baruch, Paul Slovic, and Sarah Lichtenstein. 1977. "Knowing with Certainty: The Appropriateness of Extreme Confidence." Journal of Experimental Psychology: Human Perception and Performance 3 (4): 552-64. https://doi.org/10.1037/0096-1523.3.4.552.

Hurd, Michael D. 2009. "Subjective Probabilities in Household Surveys." Annual Review of Economics 1 (June): 543-62. https://doi.org/10.1146/annurev.economics.050708.142955.

Krosnick, Jon A., Allyson L. Holbrook, Matthew K. Berent, Richard T. Carson, W. Michael Hanemann, Raymond J. Kopp, Robert Cameron Mitchell, et al. 2002. "The Impact of No Opinion Response Options on Data Quality: Non-Attitude Reduction or an Invitation to Satisfice?" Public Opinion Quarterly 66 (3): 371-403. https://doi.org/10.1086/341394. 